\title{
HOLOMORPHIC FUNCTIONS WITH DENSE SETS OF PLESSNER POINTS
}

\author{
PETER LAPPAN AND GEORGE PIRANIAN ${ }^{1}$
}

Let the function $f$ be defined in the unit disk $D=\{z:|z|<1\}$. A point $e^{i \theta}$ on the unit circle $C$ is called a Plessner point of $f$ provided each angular cluster set of $f$ at $e^{i \theta}$ coincides with the extended plane. In this note we answer the following question: if the set of Plessner points of a holomorphic function is dense on $C$, must it automatically satisfy some measure-theoretic condition?

ThEOREM. For each number $\alpha(0 \leqq \alpha \leqq 2 \pi)$ there exists a holomorphic function in $D$ whose set of Plessner points is dense on $C$ and has measure $\alpha$.

Proof. First we deal with the case where $\alpha=0$, and we begin with the construction of a locally univalent function having exactly one Plessner point.

Let $G$ be a simply connected Riemann surface, of finite area, and consisting of the unit disk together with a smooth vermiform appendage that winds its way over the plane in such a way that each point of the plane is a boundary point of $G$. Let the function $f$ map the disk $D$ conformally onto $G$, and let it map the origin onto the point $w=0$ in the portion of $G$ that covers the unit disk in the $w$-plane. Then there exists exactly one point $e^{i \theta}$ such that the image of each curve in $D$ from 0 to $e^{i \theta}$ passes through the full length of the appendage. The cluster set of $f$ relative to each curve in $D$ that terminates at $e^{i \theta}$ is the extended plane; therefore $e^{i \theta}$ is a Plessner point of $f$.

By adding further appendages to $G$, each appropriately branched, we obtain a simply connected Riemann surface $G^{*}$ such that every continuous one-to-one mapping from $D$ to $G^{*}$ has a dense set of Plessner points on $C$. If we observe the precaution of keeping the area of $G^{*}$ finite, then each conformal mapping $f^{*}$ from $D$ to $G^{*}$ has a set of Fatou points of measure $2 \pi$, hence a set of Plessner points of measure 0 .

The case where $\alpha=2 \pi$ is trivial, as we see by examining the function

$$
g(z)=\sum k_{n} z^{k_{n}}
$$

where $k_{n+1} / k_{n} \rightarrow \infty$. Since $|g(z)|>k_{n} / 4$ at all points on the circle

Received by the editors May 13, 1968 and, in revised form August 2, 1968.

1 The authors acknowledge support from the National Science Foundation. 
$|z|=1-1 / k_{n}\left(n>n_{0}\right)$, the set of Fatou points of $g$ has measure $0[2$, p. 212], and therefore its set of Plessner points has measure $2 \pi$ $[1$, p. 220].

To complete the proof, suppose that $0<\alpha<2 \pi$. Let $h$ map $D$ conformally onto the right half of the unit disk in the $w$-plane in such a way that the pre-image of the circular part of the boundary of the half-disk has length $\alpha$. Clearly, the set of Plessner points of the composite function $g^{*}=g h$ (where $g$ denotes the lacunary function in the preceding paragraph) has measure $\alpha$. Finally, let $f^{*}$ be a conformal mapping of $D$ onto $G^{*}$, and let $F=f^{*}+g^{*}$. If $e^{i \theta}$ is a Plessner point of $f^{*}$ and a Fatou point of $g^{*}$ (or vice versa), then it is a Plessner point of $F$. Therefore $F$ has the properties described in the theorem.

\section{REFERENCES}

1. A. Plessner, Über das Verhalten analytischer Funktionen am Rande ihres Definitionsbereichs, J. Reine Angew. Math. 158 (1927), 219-227.

2. L. I. Privalov, Randeigenschaften analytischer Funktionen, Deutscher Verlag Wiss., Berlin, 1956.

Michigan State University and

UNIVERSITY OF MichigAN 